\title{
A Long-Term Follow-Up Programme for Maintenance of Motor Function after Stroke: Protocol of the life after Stroke-The LAST Study
}

\author{
Torunn Askim, ${ }^{1,2}$ Birgitta Langhammer, ${ }^{1,3}$ Hege Ihle-Hansen, ${ }^{4}$ Jon Magnussen, ${ }^{2}$ \\ Torgeir Engstad, ${ }^{5}$ and Bent Indredavik ${ }^{1,6}$ \\ ${ }^{1}$ Department of Neuroscience, Faculty of Medicine, Norwegian University of Science and Technology, 7491 Trondheim, Norway \\ ${ }^{2}$ Department of Public Health and General Practice, Faculty of Medicine, Norwegian University of Science and Technology, \\ 7491 Trondheim, Norway \\ ${ }^{3}$ Physiotherapy Programme, Faculty of Health Science, Oslo and Akershus University College of Applied Sciences, 0130 Oslo, Norway \\ ${ }^{4}$ Department of Geriatric Medicine, Baerum Hospital, Vestre Viken Trust, 1309 Rud, Norway \\ ${ }^{5}$ Department of Geriatrics, University Hospital in Northern Norway, 9038 Tromsø, Norway \\ ${ }^{6}$ Stroke Unit, Department of Medicine, St. Olavs Hospital, Trondheim University Hospital, 7006 Trondheim, Norway
}

Correspondence should be addressed to Torunn Askim, torunn.askim@ntnu.no

Received 12 July 2012; Accepted 23 October 2012

Academic Editor: Susanne Palmcrantz

Copyright ( 2012 Torunn Askim et al. This is an open access article distributed under the Creative Commons Attribution License, which permits unrestricted use, distribution, and reproduction in any medium, provided the original work is properly cited.

\begin{abstract}
Background. There are no evidence-based strategies that have been shown to be superior in maintaining motor function for months to years after the stroke. The LAST study therefore intends to assess the effect of a long-term follow-up program for stroke patients compared to standard care on function, disability and health. Design. This is a prospective, multi-site randomised controlled trial, with blinded assessment 18 months after inclusion. A total of 390 patients will be recruited and randomised to a control group, receiving usual care, or to an intervention group 10 to 16 weeks after onset of stroke. Patients will be stratified according to stroke severity, age above 80 , and recruitment site. The intervention group will receive monthly coaching on physical activity by a physiotherapist for 18 consecutive months after inclusion. Outcomes. The primary outcome is motor function (Motor Assessment Scale) 18 months after inclusion. Secondary outcomes are: dependency, balance, endurance, health-related quality of life, fatigue, anxiety and depression, cognitive function, burden on caregivers, and health costs. Adverse events and compliance to the intervention will be registered consecutively during follow-up.
\end{abstract}

\section{Introduction}

Several randomised controlled trials have been performed in different countries and clinical settings to assess the effectiveness of stroke unit care for hospitalised stroke patients. The results from these studies show that stroke unit care reduces death and dependency in the short and long term [1]. The complex interventions that have shown very beneficial effects in randomised trials are also shown to be applicable and effective in routine practice [2]. After discharge from hospital a number of randomised controlled trials have shown that further follow-up by an early supported discharge service also improves independence in daily activities [3]. Despite the recommended implementation of these facilities [4], stroke still is a major cause of disability in the western countries [5]. It is hypothesised that effective interventions is needed to improve or maintain function and activity for months to years after stroke, otherwise the patient will deteriorate [4].

The effect of several more specific rehabilitation treatments during the course of stroke has also been tested, finding that repetitive task training could be beneficial to improve arm function while cardiorespiratory fitness training and task-oriented physical fitness training for walking could be beneficial to improve aspects of gait function [4]. Furthermore, the US guidelines recommend that patients 
with stroke who are capable of engaging in physical activity should do at least 30 minutes of moderate-intensity physical exercise, typically defined as vigorous activity sufficient to break a sweat or noticeable heart rate, 1 to 3 times a week [6]. However, in Norway, it is recommended that stroke patients in the chronic phase exercise at 60 to $80 \%$ of maximum heart rate for 10 to 60 minutes, 2 to 5 times a week [7]. These recommendations are largely based on expert opinions, and it appears that physical activity in community dwelling stroke survivors is far below the recommended levels [8]. Motor impairments and lack of motor skills are probably one of the most significant barriers to an active lifestyle [9]. Different community-based approaches have also been tested out in randomised controlled trials to assess the most beneficial intervention for improvement and maintenance of motor function after stroke $[10,11]$. It seems like high motivation and adherence to exercise protocols can be triggered by the test occasions and regular contact with a physiotherapist 3,6 , and 12 months after stroke, indicating that a followup program on a consultative basis is as beneficial as a compulsory exercise programme [12]. This is in contrast to the results from the ExStroke Pilot Trial showing that regular phone calls in addition to counselling of physical activity every three to six month gave no increase in physical activity compared to only counselling during the first 2 years after stroke [13]. Neither gave a lifestyle course in combination with physical activity any additional beneficial effect compared to physical activity alone [14]. There is also growing interest in health coaching as an approach to improve a healthy lifestyle in patients with chronic diseases. Health coaching includes coaching on all aspects of health such as diet, smoking habits, and physical activity and could include interventions like motivational interviews, group therapy, individual advices on life style changes, and so on. The effect is promising, however, the intervention is sparsely tested on stroke patients and more well-designed studies are warranted [15].

In summary, there are no evidence-based strategies that have been shown to be superior in maintaining motor function for months to years after the stroke [4]. Due to the increasing number of stroke patients in the next decades [16], it is a great challenge for the health care system to improve the long-term follow-up care for this group of patients and to ensure the maintenance of motor function. The maintenance must be seen as a life-long endeavour and in accordance with the need of healthy individuals to "keep in shape." Hence, a long term follow-up program including coaching of physical activity after stroke should be tested out in a randomised controlled trial. The LAST study therefore intends to assess the effect of a long term follow-up program for stroke patients compared to standard care on function, disability, and health.

The primary hypothesis is as follow.

(i) Patients receiving a long term follow-up program after stroke, comprising coaching on physical activity and exercise, will have better motor function than patients receiving standard care, 18 months after inclusion.
Secondary hypotheses are as follows.

(i) Patients receiving a long term follow-up program after stroke, comprising coaching on physical activity and exercise, will have better ADL function, better balance, better walking ability, better quality of life, less fatigue, reduced risk of new cardiovascular or cerebrovascular events and less use of health services, compared to patients receiving standard care, 18 months after inclusion.

\section{Methods}

2.1. Study Design. The LAST study is a prospective, multisite randomized controlled trial, with masked assessment at 18 months after inclusion. Patients will be enrolled at the outpatient clinic at St. Olavs Hospital or Bærum Hospital 10 to 16 weeks after onset of stroke. Figure 1 shows the study design.

2.2. Setting. Patients referred to a follow-up assessment at the outpatient clinic at one of the two study hospitals (Bærum Hospital and St. Olavs Hospital, Trondheim University Hospital) 3 months after discharge from the stroke unit will be invited to participate. The intervention will be community based and accomplished in Asker, Bærum, and Trondheim municipality. Asker and Bærum are suburbs to Oslo, having about 57000 and 114000 inhabitants, respectively, while the city of Trondheim is the third largest city in Norway with 175000 inhabitants.

2.3. Participants. All patients treated at the stroke unit at St. Olavs Hosiptal, Trondheim University Hospital and Bærum Hospital will be screened according to the following criteria at the outpatient clinic 2.5 to 4 months after onset of stroke.

\section{Inclusion Criteria:}

(i) diagnosis of stroke,

(ii) 0-4 points on Modified Rankin Scale,

(iii) mini Mental State Examination $>20$, or $>16$ for patients with aphasia,

(iv) have been discharged from hospital or inpatient rehabilitation and are community dwelling,

(v) no other serious diseases which makes it difficult to perform the intervention,

(vi) capable of providing consent.

\section{Exclusion Criteria:}

(i) seriously medical comorbidity with life expectancy $<6$ months,

(ii) medical assessment shows contraindication to participate in motor training, 


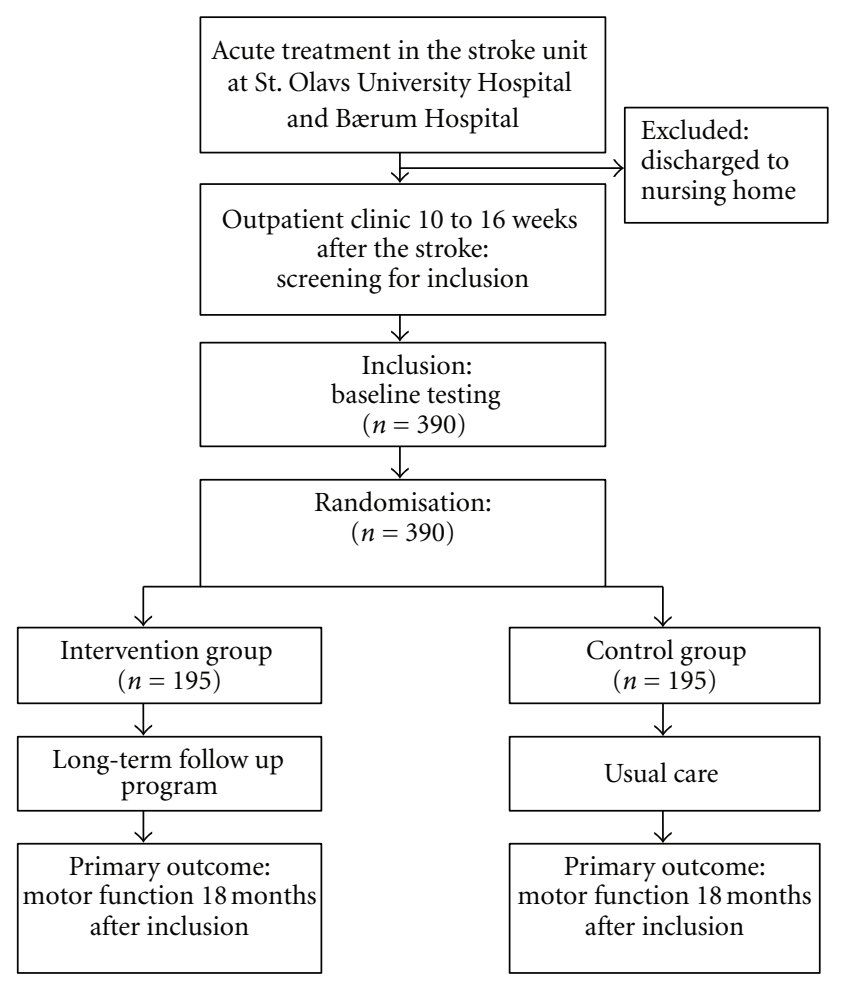

Figure 1: Study design.

(iii) other serious impairments that will have a significant impact on functional outcome,

(iv) already included in another study.

2.4. Randomisation. Participants are consecutively recruited and block randomised as they enter the study at the outpatient clinic. Patients will be stratified according to stroke severity, age above 80 , and recruitment site. The randomisation procedure will be computerised and organised by Unit for Applied Clinical Research at Norwegian University of Science and Technology (NTNU).

2.5. Baseline Data. Age, gender, marital status, pre-stroke residence, prestroke function, time for onset of stroke, time for admission to hospital, number of days in hospital/stroke unit, discharge destination, stroke severity, affected side, classification of stroke, medication, medical history, and functional state will be recorded at baseline. This information will be collected directly from the patients and/or from their hospital records. In addition, all measures listed as outcome measures will be applied at baseline.

2.6. Intervention. All patients eligible for inclusion into the LAST study will undergo evidence-based comprehensive stroke unit treatment in the acute phase [1]. Furthermore patients eligible for an early supported discharge (ESD) service [17] will be followed by a hospital based multidisciplinary team working in close cooperation with the primary health care system during the first four weeks after discharge. This follow-up care will be concluded at the outpatient clinic where the patients will be screened for inclusion into the LAST study.

2.6.1. Usual Care (Control Group). Patients randomised to usual care will be given standard community based treatment as it is given to all patients with the diagnosis of stroke within the participating municipalities. The treatment will be given as outpatient rehabilitation or as rehabilitation in the patients' home according to the patients' needs. The main approach for physiotherapists working in the participating municipalities is a nonspecialised approach, which means that they are expected to treat a range of different patient groups at older age.

2.6.2. Long-Term Follow-Up Program (Intervention Group). Patients randomised to the intervention group will in addition to usual care be given a long term follow-up program comprising a physiotherapist who will meet and coach the patients, preferably in the patients' home, on a monthly basis for 18 consecutive months after inclusion. The intervention consists of coaching on physical activity which is a significant part of health coaching. At the first meeting the patients have to complete a questionnaire about their physical activity preferences [18] to register their facilitators and barriers to physical activity. In the first meeting, all patients have to list at least one major goal for the next 3 months by use of Goal Attainment Scaling [19]. Thus, an exercise schedule in accordance with this goal and physical activity preferences will be made for the next month. The program will comprise 45-60 minutes of physical exercise once a week and 30 
minutes of physical activity every day. The weekly physical exercise will include 3 or 4 periods of rigorous activity, rated between 15 and 17 on Borgs Scale of perceived exertion. Already established community based kind of treatments will preferable be used for the rigorous exercise. Patients at the higher functional level will, if possible, participate in already established exercise groups for seniors. Patients with a lower functional level will participate in groups for people with special needs. Patients at the lowest functional level who do not fit into any group will be given individual treatment. In the following monthly meetings, the therapist and patient together will revise and agree on a new physical activity and exercise program for the next month. The patients will be responsible for executing the daily physical activity. All patients in the intervention group are asked to review their training diary on a daily basis. This is an essential part of the intervention to ensure compliance. It is also an important part of the intervention that the coaching physiotherapist will act in close cooperation with all other parts of the health care system.

2.7. Outcome Measures. Essential features of an exercise protocol for persons with stroke are motor function, activities of daily living, mobility/walking characteristics, balance, endurance, and overall health-related quality of life.

2.7.1. Primary Outcome Assessed at 18 Months after Inclusion. Motor Assessment Scale (MAS) [20] is chosen as primary outcome to assess motor function in the present trial. MAS is especially developed for persons with stroke, covering the whole range of stroke severities, and is frequently used in previous stroke trials [21]. Motor Assessment Scale is sensitive for change and the reliability and validity of the Norwegian translation of MAS is ensured [22].

2.7.2. Secondary Outcomes Assessed at 18 Months after Inclusion. Barthel Index (BI) [23] and Modified Rankin Scale (mRS) [23] are chosen to assess activities of daily living and dependency. Both scales are widely used within stroke trials. The last item of Berg Balance Scale (BBS) [24] and Timed Up and Go test (TUG) [25] are chosen to assess balance, while 10 metres maximum gait speed is chosen to assess walking ability [26]. The 6-Minute Walk Test (6MWT) is chosen to assess endurance [27]. Stroke Impact Scale (SIS) (only at 18 months) [28] and EQ-5D-5L [29] will be applied to assess health-related aspects of quality of life. Fatigue Severity Scale (FSS-7) [30] and one simple question from the HUNT3 questionnaire [31] are chosen to assess the degree of fatigue, while Hospital Anxiety and Depression Scale (HADS) [32] is chosen to assess the degree of anxiety and depression. Mini Mental State Examination (MMSE) [33] and Trailmaking $\mathrm{A}$ and $\mathrm{B}$ [34] will be applied to assess cognition. Finally Caregiver Strain Index (CSI) [35] is chosen to measure the burden of having a spouse or another significant family member suffering from stroke.

2.8. Economic Evaluation. The economic analysis will compare the costs in the long term follow-up group versus the usual care group and will involve the following.
(1) Comparison of the mean costs of therapy which will include personnel costs of community based therapy services (physiotherapy, occupational therapy, speech therapy, training groups, rehabilitation in a day clinic and day care for adults), and personnel costs of the coach/coordinating physiotherapist.

(2) Comparison of mean length of stay for patients readmitted to hospital, to inpatient rehabilitation or nursing homes.

(3) Comparison of mean costs of home-based services and mean costs of general practitioner.

Data on home based services will be collected by use of already existing computerised systems at each site, like GERICA in Trondheim and Asker and HELIOS in Bærum. In addition, data on use of private physiotherapy and general practitioners will be collected from other computerised systems. One dedicated person at each site will be responsible for collecting data on a monthly basis.

Information about readmission to hospital will also be collected by reviewing the patients hospital records every sixth month, while information about any sick leave will be collected from the Norwegian Labour and Welfare Administration (NAV).

2.9. Adverse Events. Information about death will be collected from the Norwegian Cause of Death Registry. Information about readmission to hospital and the reason for readmission like new cardiovascular or cerebrovascular events and fractures will be collected from the Norwegian Patient Registry, the Norwegian Stroke Registry and retrospectively from the patients' hospital records every sixth month.

Number of serious falls, resulting in a hospital admission or a visit at the emergency unit, will be recorded by reviewing the patients hospital record every sixth month.

2.10. Compliance. Number of completed sessions of weekly exercise and daily physical activity will be recorded in a training diary by patients randomised to the intervention group. The content, intensity, and duration of these sessions will be registered by the patient on a daily basis.

In addition, the amount of physical activity will be recorded in both groups every sixth month by use of three simple questions from the HUNT questionnaire [36] and the International Physical Activity Questionnaire (IPAQ) [37] to assess differences in activity level between the groups. In addition physical activity will be assessed by use of ActivPAL sensor system [38] for a selected group of the patients.

2.11. Sample Size. Sample size estimation was based on previous data from a comparable population [11, 39]. Ten percentage differences between the groups are considered clinically significant. The intervention group is expected to maintain their highest expected mean MAS score (38.4 points) at 18 months follow-up, while the control group is expected a $10 \%$ reduction at the same time point $(34.6$ points). The standard deviation is estimated to 10.6 points. 
Based on these assumptions, a sample size of 170 persons in each group is needed to reach a statistical power of $90 \%$. This estimate is based on alpha 0.05 . On the assumption that $15 \%$ of the participants may drop out during the course of the study a target of 390 participants has been set.

2.12. Ethics. This project will be conducted in accordance with ethical standards given by the Norwegian National Committee for Medical and Health Research Ethics. The LAST study is approved by the Regional Committee of Medical and Health Research Ethics (REC no. 2011/1427).

Only patients who are judged by a medical doctor to tolerate the intervention at the outpatient clinic will be included into the study. Patients randomised to the control group will receive today's best practice while the intervention group will get extra support from a physiotherapist in addition to today's best practice. The effect of the intervention is unknown but it is unlikely that it will increase the risk of adverse events. Hopefully it will be beneficial and improve motor function even more. As far as we can see there are no ethical second thoughts by conducting the LAST study. The LAST study is registered in ClinicalTrial.gov registry (Protocol Record 205309/H10).

2.13. Statistical Analysis. Reporting will follow the CONSORT statement for parallel group randomized trials [40]. Descriptive statistics will be performed in order to present the population and the characteristic of the two groups. All analyses will be analysed as intention-to-treat analysis according to the CONSORT instructions. Analysis of covariance (ANCOVA) will be used to study differences between groups according to the primary endpoint, motor assessment scale at 18 months after inclusion. The ANCOVA model will include stroke severity, age, pre stroke disability, treatment group, and motor function at baseline as covariates. MannWhitney $U$ test will be used for secondary data not being normal distributed. In all analyses we will control for potential confounding factors, investigate effect modifications, and present both unadjusted and adjusted effects with 95\% confidence intervals.

Subanalysis will be performed according to the stratification variables (stroke severity, age above 80 and recruitment site) to explore trends within subgroups of patients.

2.14. Recruitment. Recruitment to the trial commenced in November 2011. By July 2012, 100 patients had been randomised.

\section{Summary}

It is shown that repetitive task training is beneficial to improve motor function in all phases after stroke. Furthermore, it is recommended that stroke patients engage in physical activity and exercise up to 5 days a week to maintain motor function and reduce the risk of recurrent strokes. Even though these recommendations are largely based on expert opinions, the maintenance must be seen as a lifelong endeavour and in accordance with the need of healthy individuals to "keep in shape." Motor impairments are one of the barriers for stroke patients to comply with these recommendations. Hence, there is a need for strategies to facilitate motor training and maintain motor function at a high level in the long term and thereby reduce the need for health services and reduce the health costs after stroke.

Health coaching includes coaching on all aspects of health such as diet, smoking habits, and physical activity. Health coaching might improve a healthy lifestyle in patients with chronic diseases and could include interventions like motivational interviews, group therapy, individual advices on life style changes, and so on. The effect seems promising, however, it is not widely tested on patients after stroke.

In a randomised controlled trial, we want to test the effect of coaching on physical activity performed by a physiotherapist aiming to maintain motor function in the long run after stroke. The coaching will be given in monthly meetings for 18 consecutive months after inclusion. In the first meeting facilitators and barriers to physical activity will be registered by use of a physical activity preferences questionnaire and the patients' individual goals will be registered by use of Goal Attainment Scaling. Based on this information, a physical activity and exercise program for the next month will be set up. The program will comprise at least 45-60 minutes of physical exercise once a week and 30 minutes of physical activity every day. Already established community based kind of treatments will preferablly be used for the rigorous exercise. The patients will be responsible for executing the daily physical activity. To ensure compliance, all patients in the intervention group have to review their training diary on a daily basis.

In addition to the potential to improve functional outcomes, the LAST study has the potential to reduce the health costs by reducing the number of readmissions to hospital and reducing the need for home care services. Stroke patients are at increased risk of immobility-related complications increasing the risk of readmission to hospital. Hence, a small increase in activity level will reduce this risk and save health money for alternative use.

With the aging population and the rising number of stroke patients, the outcome of this trial has the potential to benefit a large section of the population both in Norway and in other western countries. The intervention will be tested out in three different community based settings and if it shows to be beneficial, it will be easy to adjust and implement into several other different kind of community based settings. If the results turn out to be neutral or negative, we have the scientific evidence that usual care still is the most beneficial approach.

\section{Study Organisation and Funding}

This project is organised at the Norwegian University of Science and Technology (NTNU), Faculty of Medicine, Department of Neuroscience. A project group and a steering committee have been set down to manage the project. The LAST study is funded by the Norwegian Research Council, the Collaboration Committee between Central Norway 
Health Authority, and Norwegian University of Science and Technology and by grants from the Stroke Unit Research Fund.

\section{Details of the LAST Study Group}

The Steering Committee is composed of: Professor Bent Indredavik, Associate Professor Torunn Askim, Associate Professor Birgitta Langhammer, Associate Professor Torgeir Engstad, Professor Jon Magnussen, MD Hege Ihle-Hansen, Anne Hansen (Head of Department of Physiotherapy, City of Trondheim, Norway), Rigmor Rytter Axelsen (Head of Department of Rehabilitation, municipality of Bærum, Norway), Rune Schjørlien (Head of Department of Prevention and Rehabilitation, municipality of Asker, Norway), and Randi Moxnes Osmundnes (Landsforeningen for slagrammede, Norway). International collaborating partners are: Professor Peter Langhorne (University of Glasgow, UK), Associate Professor Julie Bernhardt (Florey Neuroscience Institutes, Melbourne, Australia).

\section{References}

[1] Stroke Unit Trialists' Collaboration, "Organised inpatient (stroke unit) care for stroke," Cochrane Database of Systematic Reviews, no. 4, Article ID CD000197, 2007.

[2] P. Seenan, M. Long, and P. Langhorne, "Stroke units in their natural habitat: systematic review of observational Studies," Stroke, vol. 38, no. 6, pp. 1886-1892, 2007.

[3] P. Langhorne, G. Taylor, G. Murray et al., "Early supported discharge services for stroke patients: a meta-analysis of individual patients' data," The Lancet, vol. 365, no. 9458, pp. 501-506, 2005.

[4] P. Langhorne, J. Bernhardt, and G. Kwakkel, "Stroke rehabilitation," The Lancet, vol. 377, no. 9778, pp. 1693-1702, 2011.

[5] A. D. Lopez, C. D. Mathers, M. Ezzati, D. T. Jamison, and C. J. Murray, "Global and regional burden of disease and risk factors, 2001: systematic analysis of population health data," The Lancet, vol. 367, no. 9524, pp. 1747-1757, 2006.

[6] K. L. Furie, S. E. Kasner, R. J. Adams et al., "Guidelines for the prevention of stroke in patients with stroke or transient ischemic attack: a guideline for healthcare professionals from the American heart association/American stroke association," Stroke, vol. 42, no. 1, pp. 227-276, 2011.

[7] Aktivitetshåndboken, "Fysisk aktivitet i forebygging og behandling," http://helsedirektoratet.no/folkehelse/fysisk-aktivitet/ aktivitetshandboken/Sider/default.aspx.

[8] D. Rand, J. J. Eng, P. F. Tang, J. S. Jeng, and C. Hung, "How active are people with stroke? Use of accelerometers to assess physical activity," Stroke, vol. 40, no. 1, pp. 163-168, 2009.

[9] K. R. Zalewski and L. Dvorak, "Barriers to physical activity between adults with stroke and their care partners," Topics in Stroke Rehabilitation, vol. 18, supplement 1, pp. 666-675, 2011.

[10] B. Langhammer, B. Lindmark, and J. K. Stanghelle, "Stroke patients and long-term training: is it worthwhile? A randomized comparison of two different training strategies after rehabilitation," Clinical Rehabilitation, vol. 21, no. 6, pp. 495510, 2007.

[11] T. Askim, S. Mørkved, A. Engen, K. Roos, T. Aas, and B. Indredavik, "Effects of a community-based intensive motor training program combined with early supported discharge after treatment in a comprehensive stroke unit: a randomized, controlled trial," Stroke, vol. 41, no. 8, pp. 1697-1703, 2010.

[12] B. Langhammer, J. K. Stanghelle, and B. Lindmark, "Exercise and health-related quality of life during the first year following acute stroke. A randomized controlled trial," Brain Injury, vol. 22, no. 2, pp. 135-145, 2008.

[13] G. Boysen, L. H. Krarup, X. Zeng et al., "ExStroke pilot trial of the effect of repeated instructions to improve physical activity after ischaemic stroke: a multinational randomised controlled clinical trial," British Medical Journal, vol. 339, Article ID b2810, 2009.

[14] A. Lund, M. Michelet, L. Sandvik, T. Wyller, and U. Sveen, "A lifestyle intervention as supplement to a physical activity programme in rehabilitation after stroke: a randomized controlled trial," Clinical Rehabilitation, vol. 26, no. 6, pp. 502$512,2012$.

[15] J. M. Olsen and B. J. Nesbitt, "Health coaching to improve healthy lifestyle behaviors: an integrative review," American Journal of Health Promotion, vol. 25, no. 1, pp. e1-e12, 2010.

[16] H. Waaler, Scenario 2030: Sykdomsutvikling for eldre fram til 2030, Statens helsetilsyn, Oslo, Norway, 1999.

[17] Early Supported Discharge Trialists, "Services for reducing duration of hospital care for acute stroke patients," Cochrane Database of Systematic Reviews, no. 2, Article ID CD000443, 2007.

[18] G. Banks, J. Bernhardt, L. Churilov, and T. B. Cumming, "Exercise preferences are different after stroke," Stroke Research and Treatment, vol. 2012, Article ID 890946, 9 pages, 2012.

[19] K. Rockwood, P. Stolee, and R. A. Fox, "Use of goal attainment scaling in measuring clinically important change in the frail elderly," Journal of Clinical Epidemiology, vol. 46, no. 10, pp. 1113-1118, 1993.

[20] J. H. Carr, R. B. Shepherd, L. Nordholm, and D. Lynne, "Investigation of a new motor assessment scale for stroke patients," Physical Therapy, vol. 65, no. 2, pp. 175-180, 1985.

[21] B. French, L. H. Thomas, M. J. Leathley et al., "Repetitive task training for improving functional ability after stroke," Cochrane Database of Systematic Reviews, no. 4, Article ID CD006073, 2007.

[22] A. Kjendahl, R. Jahnsen, and G. Aamodt, "Motor assessment scale, oversettelse til norsk og inter-rater reliabilitet," Fysioterapeuten, no. 5, pp. 14-18, 2005.

[23] C. D. A. Wolfe, N. A. Taub, E. J. Woodrow, and P. G. J. Burney, "Assessment of scales of disability and handicap for stroke patients," Stroke, vol. 22, no. 10, pp. 1242-1244, 1991.

[24] L. Alzayer, M. Beninato, and L. G. Portney, "The accuracy of individual berg balance scale items compared with the total berg score for classifying people with chronic stroke according to fall history," Journal of Neurologic Physical Therapy, vol. 33, no. 3, pp. 136-143, 2009.

[25] S. Mathias, U. S. L. Nayak, and B. Isaacs, "Balance in elderly patients: the "get-up and go" test," Archives of Physical Medicine and Rehabilitation, vol. 67, no. 6, pp. 387-389, 1986.

[26] R. W. Bohannon, "Comfortable and maximum walking speed of adults aged 20-79 years: reference values and determinants," Age and Ageing, vol. 26, no. 1, pp. 15-19, 1997.

[27] G. H. Guyatt, M. J. Sullivan, P. J. Thompson, E. L. Fallen, S. O. Pugsley, D. W. Taylor et al., "The 6-minute walk: a new measure of exercise capacity in patients with chronic heart failure," Canadian Medical Association Journal, vol. 132, no. 8, pp. 919-923, 1985.

[28] P. W. Duncan, D. Wallace, S. M. Lai, D. Johnson, S. Embretson, and L. J. Laster, "The stroke impact scale version 2.0: 
evaluation of reliability, validity, and sensitivity to change," Stroke, vol. 30, no. 10, pp. 2131-2140, 1999.

[29] R. Rabin and F. de Charro, "EQ-5D: a measure of health status from the EuroQol Group," Annals of Medicine, vol. 33, no. 5, pp. 337-343, 2001.

[30] A. Lerdal, A. Kottorp, C. Gay, B. E. Aouizerat, C. J. Portillo, and K. A. Lee, "A 7-item version of the fatigue severity scale has better psychometric properties among HIV-infected adults: an application of a Rasch model," Quality of Life Research, vol. 20, no. 9, pp. 1447-1456, 2011.

[31] "The HUNT study-a longitudinal population health study in Norway," HUNT3-Q1-all participants age 20 and older, men and women, 2012, http://www.ntnu.edu/hunt/data/que.

[32] A. S. Zigmond and R. P. Snaith, "The hospital anxiety and depression scale," Acta Psychiatrica Scandinavica, vol. 67, no. 6, pp. 361-370, 1983.

[33] M. F. Folstein, S. E. Folstein, and P. R. McHugh, "'Mini mental state. A practical method for grading the cognitive state of patients for the clinician," Journal of Psychiatric Research, vol. 12, no. 3, pp. 189-198, 1975.

[34] T. N. Tombaugh, "Trail making test A and B: normative data stratified by age and education," Archives of Clinical Neuropsychology, vol. 19, no. 2, pp. 203-214, 2004.

[35] B. C. Robinson, "Validation of a caregiver strain index," Journals of Gerontology, vol. 38, no. 3, pp. 344-348, 1983.

[36] N. Kurtze, V. Rangul, B. E. Hustvedt, and D. W. Flanders, "Reliability and validity of self-reported physical activity in the Nord-Trøndelag health study-HUNT 1," Scandinavian Journal of Public Health, vol. 36, no. 1, pp. 52-61, 2008.

[37] C. L. Craig, A. L. Marshall, M. Sjöström et al., "International physical activity questionnaire: 12-country reliability and validity," Medicine and Science in Sports and Exercise, vol. 35, no. 8, pp. 1381-1395, 2003.

[38] K. Taraldsen, T. Askim, O. Sletvold et al., "Evaluation of a body-worn sensor system to measure physical activity in older people with impaired function," Physical Therapy, vol. 91, no. 2, pp. 277-285, 2011.

[39] B. Langhammer, J. K. Stanghelle, and B. Lindmark, "An evaluation of two different exercise regimes during the first year following stroke: a randomised controlled trial," Physiotherapy Theory and Practice, vol. 25, no. 2, pp. 55-68, 2009.

[40] K. F. Schulz, D. G. Altman, and D. Moher, "CONSORT 2010 statement: Updated guidelines for reporting parallel group randomized trials," Annals of Internal Medicine, vol. 152, no. 11, pp. 726-732, 2010. 


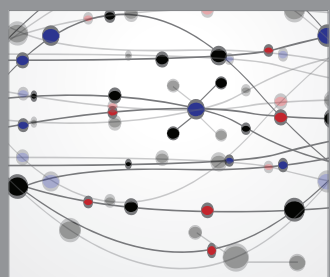

The Scientific World Journal
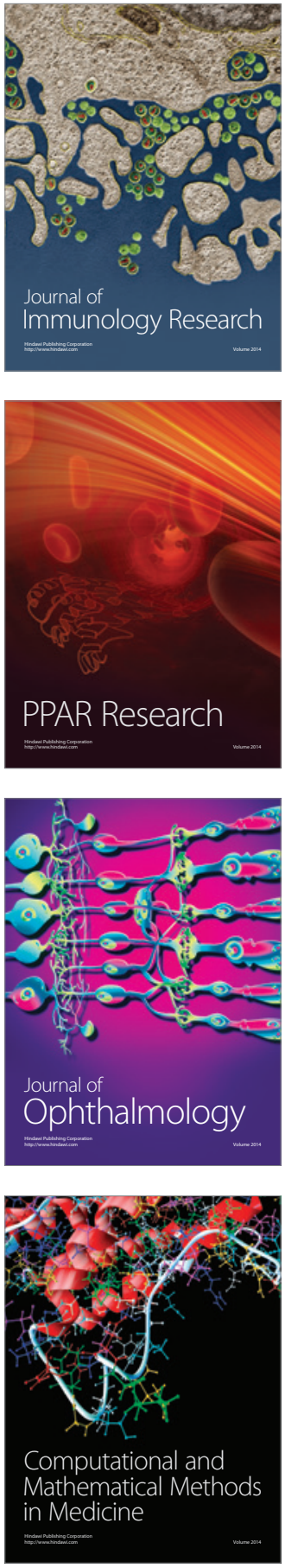

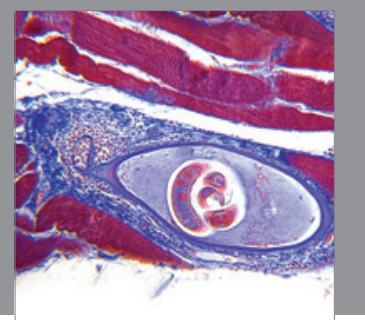

Gastroenterology

Research and Practice
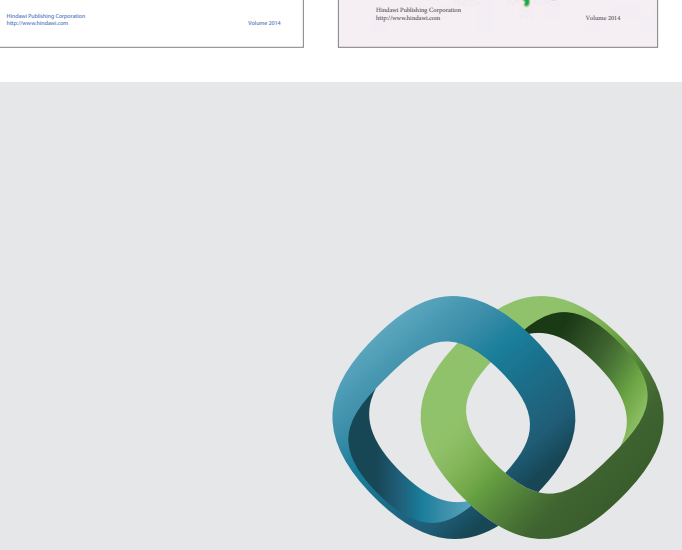

\section{Hindawi}

Submit your manuscripts at

http://www.hindawi.com
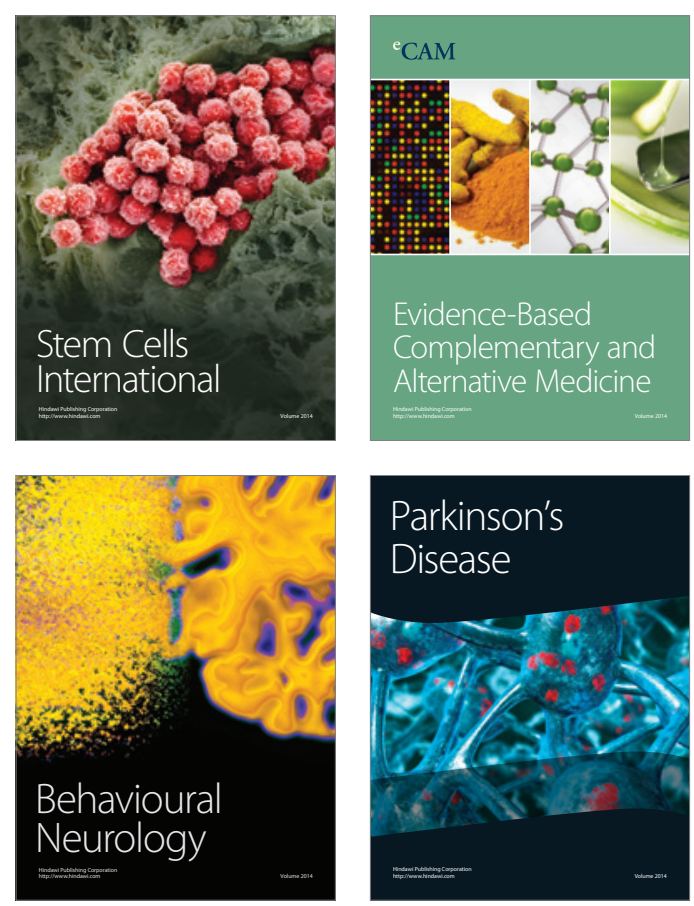

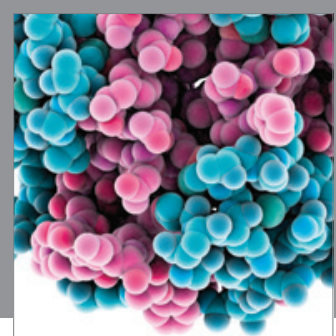

Journal of
Diabetes Research

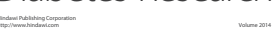

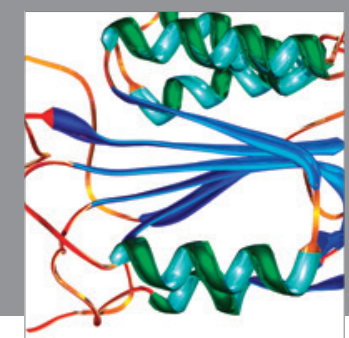

Disease Markers
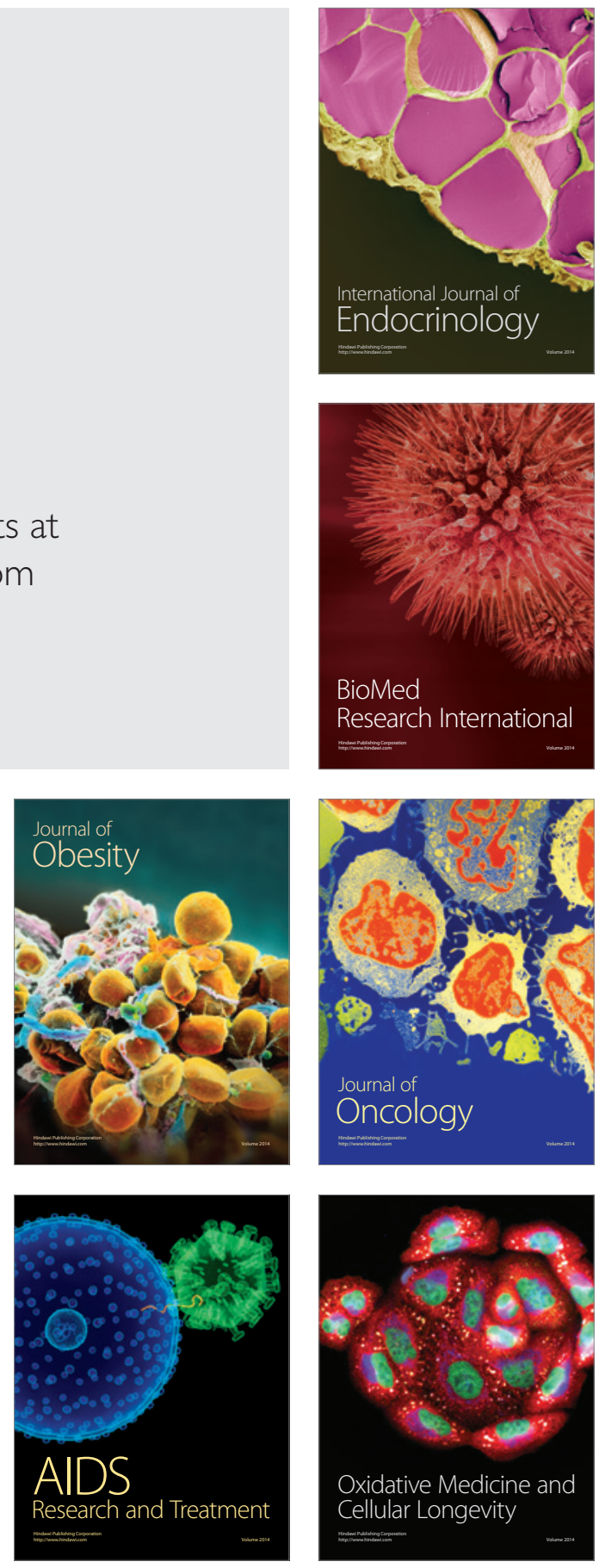